\title{
Survival, Prognostic Factors, and Volumetric Analysis of Extent of Resection for Anaplastic Gliomas
}

\author{
Je Beom Hong, MD ${ }^{1,2}$ \\ Tae Hoon Roh, MD, $\mathrm{PhD}$ \\ Seok-Gu Kang, MD, PhD 24,5 \\ Se Hoon Kim, MD, PhD ${ }^{4,6}$ \\ Ju Hyung Moon, MD2,4 \\ Eui Hyun Kim, MD, PhD2,45 \\ Sung Soo Ahn, MD, PhD ${ }^{4,7}$ \\ Hye Jin Choi, MD, $\mathrm{PhD}{ }^{4.8}$ \\ Jaeho Cho, MD, PhD ${ }^{4,9}$ \\ Chang-Ok Suh, MD, PhD ${ }^{4,9}$ \\ Jong Hee Chang, MD, PhD 2,45
}

${ }^{1}$ Department of Neurosurgery,

Kangbuk Samsung Hospital, Sungkyunkwan

University School of Medicine, Seoul,

${ }^{2}$ Department of Neurosurgery,

Yonsei University College of Medicine, Seoul,

${ }^{3}$ Department of Neurosurgery,

Ajou University Hospital, Ajou University

School of Medicine, Suwon, ${ }^{4}$ Brain Tumor

Center, Severance Hospital,

Yonsei University Health System, Seoul,

${ }^{5}$ Brain Research Institute,

Yonsei University College of Medicine,

Seoul, Departments of ${ }^{6}$ Pathology,

${ }^{7}$ Radiology, ${ }^{8}$ Division of Oncology,

Department of Internal Medicine,

and ${ }^{9}$ Department of Radiation Oncology,

Yonsei University College of Medicine,

Seoul, Korea

Correspondence: Jong Hee Chang, MD, PhD

Department of Neurosurgery, Yonsei University

College of Medicine, 50-1 Yonsei-ro,

Seodaemun-gu, Seoul 03722, Korea

Tel: 82-2-2228-2162

Fax: 82-2-393-9979

E-mail: changih@yuhs.ac

Received January 23, 2020

Accepted April 22, 2020

Published Online April 23, 2020

\section{Purpose}

The aim of this study is to evaluate the survival rate and prognostic factors of anaplastic gliomas according to the 2016 World Health Organization classification, including extent of resection (EOR) as measured by contrast-enhanced T1-weighted magnetic resonance imaging (MRI) and the T2-weighted MRI.

\section{Materials and Methods}

The records of 113 patients with anaplastic glioma who were newly diagnosed at our institute between 2000 and 2013 were retrospectively reviewed. There were 62 cases (54.9\%) of anaplastic astrocytoma, isocitrate dehydrogenase (IDH) wild-type (AAw), 18 cases (16.0\%) of anaplastic astrocytoma, IDH-mutant, and 33 cases (29.2\%) of anaplastic oligodendroglioma, IDH-mutant and 1p/19q-codeleted.

\section{Results}

The median overall survival (OS) was 48.4 months in the whole anaplastic glioma group and 21.5 months in AAw group. In multivariate analysis, age, preoperative Karnofsky Performance Scale score, $0^{6}$-methylguanine-DNA methyltransferase (MGMT) methylation status, postoperative tumor volume, and EOR measured from the T2 MRI sequence were significant prognostic factors. The EOR cut-off point for OS measured in contrast-enhanced T1-weighted MRI and T2-weighted MRI were 99.96\% and 85.64\%, respectively.

\section{Conclusion}

We found that complete resection of the contrast-enhanced portion (99.96\%) and more than $85.64 \%$ resection of the non-enhanced portion of the tumor have prognostic impacts on patient survival from anaplastic glioma.

\section{Key words}

Anaplastic glioma, Extent of resection, Survival, Prognosis

\section{Introduction}

Anaplastic gliomas, which account for 15\%-20\% of malignant gliomas [1], have poor prognosis despite modern mul- timodal treatments. To improve the accuracy of diagnosis and treatment, the 2016 World Health Organization (WHO) classification changed the three original categories from the 2007 classification, namely anaplastic astrocytoma, anaplastic 
oligodendroglioma, and anaplastic oligoastrocytoma. These three categories were further subdivided as follows: anaplastic astrocytoma, isocitrate dehydrogenase (IDH)-mutant (AAm); anaplastic astrocytoma, IDH-wildtype (AAw); anaplastic astrocytoma, not otherwise specified (NOS); anaplastic oligodendroglioma, IDH-mutant and 1p/19q-codeleted (AOmc); anaplastic oligodendroglioma, NOS; and anaplastic oligoastrocytoma, NOS [2]. We obtained survival rates according to the new classification and examined the associated prognostic factors.

Extent of resection (EOR) has been known as an important prognosticator in anaplastic gliomas [3,4]. However, many studies have focused on only the contrast-enhanced parts of tumors observed in T1-weighted magnetic resonance imaging (MRI), despite many cases of anaplastic gliomas that are not enhanced or are only partially enhanced in contrastenhanced T1-weightedimages. In thepresentstudy, weinvestigated the tumor volume and EOR in both contrast-enhanced T1-weighted MRI and T2-weighted MRI sequences.

The aims of this study were (1) to identify the survival rate and prognostic factors in patients with anaplastic gliomas in the 2016 WHO classification; (2) to determine whether the volumetric measurement of EOR has prognostic value in contrast-enhanced T1-weighted MRI and T2-weighted MRI, and (3) to determine the prognostically meaningful cut-off value of resection volume in each MRI sequence.

\section{Materials and Methods}

\section{Patient selection}

We performed a retrospective analysis of the medical records and MRI features of 113 consecutive patients with anaplastic glioma who were newly diagnosed at our institute between 2000 and 2013, with neither prior radiotherapy nor chemotherapy. We excluded patients with gliomatosis cerebri (involving more than three lobes), midline location, and malignant transformation of a previously operated low-grade glioma. The medical records reviewed included sex, age at first diagnosis, preoperative Karnofsky Performance Scale (KPS) score, and postoperative treatment (radiotherapy, chemotherapy) (Table 1 ).

\section{Histopathologic review}

We investigated the molecular profiles of all patients including $1 \mathrm{p} / 19 \mathrm{q}$ codeletion status, methylation of the $\mathrm{O}^{6}$-methylguanine-DNA methyltransferase (MGMT) promoter and the state of $I D H$ mutation. The IDH mutation status was initially assessed using immunostaining for the IDH1-R132H mutation. If immunohistochemistry did not show a mutation in IDH1-R132H, sequencing of IDH1 codon 123 and IDH2 codon 172 was performed. The 113 cases (grade III glioma based on the 2007 classification) were re-classified using the
2016 classification system: cases with wild-type IDH were classified as AAw, cases with non-codeleted $1 \mathrm{p} / 19 \mathrm{q}$ and mutated IDH were classified as AAm, and cases with an IDH mutation and $1 \mathrm{p} / 19 \mathrm{q}$ codeletion were classified as AOmc. This re-classification identified 62 cases of AAw, 18 cases of AAm, and 33 cases of AOmc. All pathological and molecular data were reviewed by a single pathologist (S.H.K.).

\section{Imaging evaluation}

MRI sequences, including T2-weighted, fluid-attenuated inversion recovery (FLAIR) and contrast-enhanced T1-weighted, were obtained preoperatively, postoperatively, and at regular follow-ups. Two experienced radiologists reviewed all patients' MRI data. Manual segmentation was performed to measure the tumor and resection volumes. We used OsiriX software (Pixmeo SARL, Bernex, Switzerland) to measure tumor volumes and EOR. Tumor volume was estimated based on the area of increased signal intensity on the contrast-enhanced T1-weighted images (enhancing lesions) or T2-weighted images (non-enhancing lesions). We tried to exclude any regions with cerebral edema on the T2-weighted images. The non-enhancing tumor was defined with regions of T2 hyperintensity (less than cerebrospinal fluid signal) that were associated with mass effect and architectural distortion, including blurring of the gray matter/white matter interface. Edema needed to be greater in signal than the non-enhancing tumor and lower than the cerebrospinal fluid on T2. The resection extent was calculated using early postoperative images ( $<48$ hours). EOR was calculated with the following equation: (preoperative tumor volume-postoperative tumor volume)/ preoperative tumor volume. With respect to tumor location, deep lesions were defined as those that involved the brainstem, thalamus, basal ganglia, and the insula; superficial lesions involved only the cortex outside the insula.

\section{Statistical analyses}

We analyzed overall survival (OS) and progression-free survival (PFS) according to the specific pathology type using Kaplan-Meier curves and log-rank tests. To identify factors associated with PFS and OS, univariate and a multivariate Cox proportional regression analyses with stepwise methods (entry and exit criteria of $\mathrm{p}<0.05$ ) were performed, using the time from surgery to progression or death. Age, preoperative KPS score, tumor volume, and EOR were evaluated as continuous variables. OS was defined as the time from surgery to death from any cause or the last follow-up. PFS was defined as the time from surgery to the first instance of radiological signs of progression and/or deteriorated neurological status or death. We estimated optimal cut-off values for the dichotomization of the clinical outcome variable based on time-to-event data using the technique devised by Contal and O'Quigley [5]. The optimal cut-off point was selected by maximizing the hazard ratio. $\mathrm{p}$-value $<0.05$ was considered 
Table 1. Baseline patient characteristics

\begin{tabular}{|c|c|c|c|c|}
\hline & AAw & AAm & AOmc & Total \\
\hline No. of patients & $62(54.9)$ & $18(16.0)$ & $33(29.2)$ & $113(100)$ \\
\hline Age, median (range, yr) & $48(18-82)$ & $36(18-71)$ & $45(24-76)$ & $40(18-82)$ \\
\hline Sex, female/male & $27 / 35$ & $8 / 10$ & $14 / 19$ & $49 / 64$ \\
\hline Preoperative KPS, median (range) & $80(40-90)$ & $80(80-90)$ & $80(70-100)$ & $80(40-100)$ \\
\hline \multicolumn{5}{|l|}{ Postoperative treatment } \\
\hline CCRT & $5(4.4)$ & $1(0.9)$ & 0 & $6(5.3)$ \\
\hline $\mathrm{CCRT} \rightarrow \mathrm{CT}$ & $15(13.3)$ & $1(0.9)$ & $9(8.0)$ & $25(22.1)$ \\
\hline $\mathrm{RT} \rightarrow \mathrm{CT}$ & $12(10.6)$ & $7(6.2)$ & $9(8.0)$ & $28(24.8)$ \\
\hline RT & $20(17.7)$ & $8(7.1)$ & $15(13.3)$ & $43(38.1)$ \\
\hline None & $4(3.5)$ & 0 & 0 & $4(3.5)$ \\
\hline Unknown & $6(5.3)$ & $1(0.9)$ & 0 & $7(6.2)$ \\
\hline \multicolumn{5}{|l|}{ Chemotherapy regimen } \\
\hline PCV & $9(8.0)$ & $1(0.9)$ & $5(4.4)$ & $15(13.3)$ \\
\hline TMZ & $21(18.6)$ & $8(7.1)$ & $12(10.6)$ & $41(36.3)$ \\
\hline Others ${ }^{\text {a) }}$ & $2(1.8)$ & 0 & $1(0.9)$ & $3(2.7)$ \\
\hline \multicolumn{5}{|l|}{ MGMT promoter status } \\
\hline Methylated & $20(17.7)$ & $15(13.3)$ & $29(25.7)$ & $64(56.6)$ \\
\hline Unmethylated & $41(36.3)$ & $3(2.7)$ & $4(3.5)$ & $48(42.5)$ \\
\hline Missing & $1(0.9)$ & 0 & 0 & $1(0.9)$ \\
\hline \multicolumn{5}{|l|}{ Tumor location } \\
\hline Deep & $24(21.2)$ & $4(3.5)$ & $5(4.4)$ & $33(29.2)$ \\
\hline Superficial & $32(28.3)$ & $12(10.6)$ & $26(23.0)$ & $70(61.9)$ \\
\hline \multicolumn{5}{|l|}{ Volumetric analysis, mean (range) } \\
\hline Preoperative (T1CE, $\mathrm{cm}^{3}$ ) & $14.6(0.0-117.8)$ & $3.2(0.0-14.8)$ & $18.0(0-112.2)$ & $13.8(0.0-117.8)$ \\
\hline Preoperative $\left(\mathrm{T} 2, \mathrm{~cm}^{3}\right)$ & $48.7(0.8-197.2)$ & $83.9(13.5-232.9)$ & $87.1(6.2-212.3)$ & $51.6(0.8-232.9)$ \\
\hline Postoperative (T1CE, $\mathrm{cm}^{3}$ ) & $2.62(0-24.4)$ & $0.0(0-0)$ & $0.1(0-2.5)$ & $1.0(0-24.4)$ \\
\hline Postoperative $\left(\mathrm{T} 2, \mathrm{~cm}^{3}\right)$ & $19.3(0-166.5)$ & $4.9(0.7-27.3)$ & $4.3(0-26.9)$ & $9.9(0-166.5)$ \\
\hline Extent of resection $(\mathrm{T} 1 \mathrm{CE}, \%)$ & $81.5(0-100)$ & $100.0(100-100)$ & $95.9(41.2-100)$ & $90.6(0-100)$ \\
\hline Extent of resection $(\mathrm{T} 2, \%)$ & $75.4(0-100)$ & $92.5(52.7-100)$ & $95.4(76.5-100)$ & $87.4(0-100)$ \\
\hline
\end{tabular}

Values are presented as number (\%) unless otherwise indicated. AAw, anaplastic astrocytoma, IDH-wildtype; AAm, anaplastic astrocytoma, IDH-mutant; AOmc, anaplastic oligodendroglioma, IDH-mutant, and 1p/19q-codeleted; KPS, Karnofsky Performance Scale; CCRT, concurrent chemo-radiation therapy; $\mathrm{CT}$, chemotherapy; $\mathrm{RT}$, radiation therapy; PCV, procarbazine, lomustine, and vincristine; TMZ, temozolomide; MGMT, $\mathrm{O}^{6}$-methylguanine-DNA methyltransferase; T1CE, T1-weighted contrast-enhanced magnetic resonance imaging (MRI); T2, T2-weighted MRI. a) Others included fluorouracil +carboplatin, vincristine, and lomustine.

statistically significant. All statistical procedures were performed using SAS for Windows ver. 9.4 (SAS Institute Inc., Cary, NC).

\section{Ethical statement}

All methods were performed in accordance with the ethical guidelines of the 1975 Deceleration of Helsinki, as revised in 1983, and was approved by the institutional review board of Severance Hospital (Yonsei University Health System, Severance Hospital, 4-2019-0181). The written informed consent was waived by the institutional review board that approved this study's protocol because all the information was tabulated in anonymized and de-identified fashion.

\section{Results}

\section{Patient characteristics}

The clinical information of all 113 patients are listed in Table 1, stratified by the 2016 WHO classification. There were 62 patients (54.9\%) in the AAw group, $18(16.0 \%)$ in the AAm group, and $33(29.2 \%)$ in the AOmc group. The median age at first diagnosis in the whole cohort was 40 years (range, 18 to 82 years). In total, 102 patients $(90.3 \%)$ received postoperative radiotherapy and $59(52.2 \%)$ received chemotherapy. Among the chemotherapy regimens, 15 cases $(13.3 \%)$ were treated with PCV (procarbazine, lomustine, and vincristine) and $41(36.3 \%)$ with temozolomide. The postoperative treatment modalities are also presented in Table 1. MGMT promoter methylation was detected in 64 cases $(56.6 \%)$ of the 
Table 2. Overall survival (OS) and progression-free survival (PFS)

\begin{tabular}{|c|c|c|c|c|c|c|}
\hline \multirow{2}{*}{ Group } & \multirow{2}{*}{ Median (mo) } & \multicolumn{5}{|c|}{ Survival rate $(\%)$} \\
\hline & & 1-Year & 2-Year & 3-Year & 4-Year & 5-Year \\
\hline \multicolumn{7}{|l|}{ OS } \\
\hline GIII all & 48.4 & 84.8 & 64.9 & 55.6 & 50.7 & 45.3 \\
\hline AAw & 21.5 & 74.1 & 64.0 & 46.6 & 28.3 & 14.4 \\
\hline AAm & n.r. & 69.3 & 55.4 & 55.4 & 55.4 & 55.4 \\
\hline AOmc & n.r. & 96.3 & 85.9 & 85.9 & 85.9 & 85.9 \\
\hline \multicolumn{7}{|l|}{ PFS } \\
\hline GIII all & 31.8 & 76.7 & 58.7 & 49.2 & 43.1 & 41.8 \\
\hline AAw & 16.4 & 80.6 & 64.5 & 45.7 & 25.8 & 9.0 \\
\hline AAm & n.r. & 88.9 & 83.0 & 83.0 & 62.2 & 62.2 \\
\hline $\mathrm{AOmc}$ & 130.0 & 96.4 & 92.4 & 82.6 & 82.6 & 82.6 \\
\hline
\end{tabular}

GIII, grade III glioma; AAw, anaplastic astrocytoma, IDH-wildtype; AAm, anaplastic astrocytoma, IDH-mutant; n.r., not reached; AOmc, anaplastic oligodendroglioma, IDH-mutant and 1p/19q-codeleted.

A
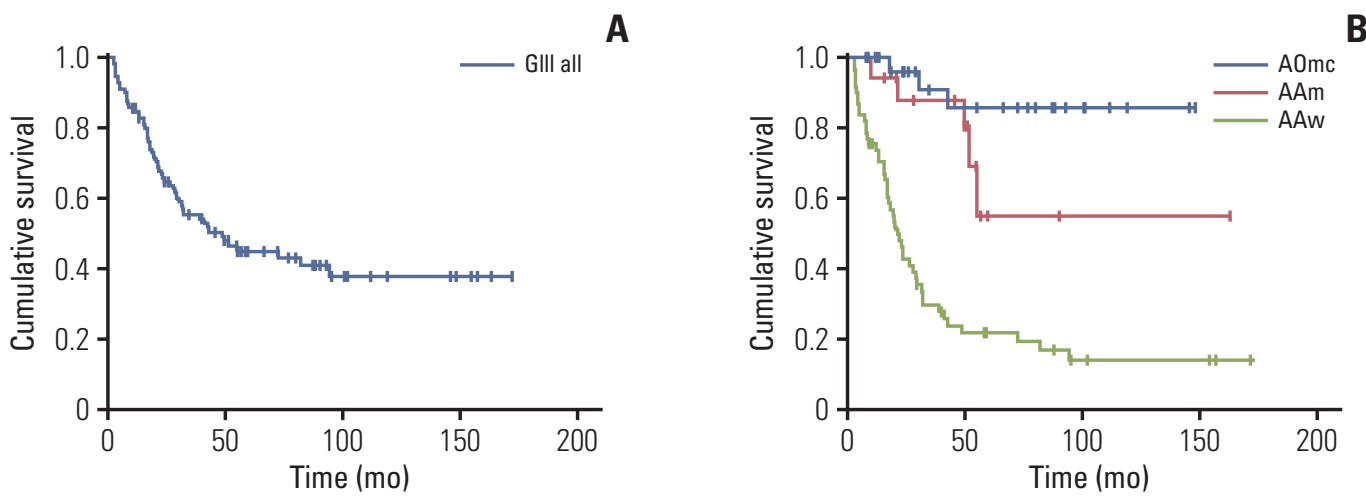

Fig. 1. Overall survival. (A) Kaplan-Meier representation of overall survival time for the entire group of 113 patients. (B) Kaplan-Meier representation of overall survival time for the AAw, AAm, AOmc each group. AAm, anaplastic astrocytoma, IDH-mutant; AAw, anaplastic astrocytoma, IDH-wildtype; AOmc, anaplastic oligodendroglioma, IDH-mutant and 1p/19q-codeleted; GIII, grade III glioma.

whole cohort, 20 cases $(17.7 \%)$ in the AAw subgroup, 15 cases $(13.3 \%)$ in the AAm subgroup, and 29 cases $(25.7 \%)$ in the AOmc subgroup.

\section{Volumetric analysis}

The mean tumor volumes in the contrast-enhanced T1weighted and T2-weighted MRI sequences were respectively $13.8 \mathrm{~cm}^{3}$ (range, 0.0 to $117.8 \mathrm{~cm}^{3}$ ) and $51.6 \mathrm{~cm}^{3}$ (range, 0.8 to $232.9 \mathrm{~cm}^{3}$ ) preoperatively, and $1.0 \mathrm{~cm}^{3}$ (range, 0.0 to 24.4 $\mathrm{cm}^{3}$ ) and $9.9 \mathrm{~cm}^{3}$ (range, 0.0 to $166.5 \mathrm{~cm}^{3}$ ) postoperatively. The EOR measured in contrast-enhanced T1-weighted MRI (EOR-T1CE) was $90.6 \%$ (range, $0 \%$ to $100 \%$ ), while it was $87.4 \%(0.0 \%-100 \%)$ in the T2-weighted MRI sequence. In our study biopsies were performed in 25 patients (22.1\%).

\section{Survival}

After a median follow-up period of 66.1 months, the median OS was 48.4 months (95\% confidence interval [CI], 15.1 to 81.7$)$ for all patients and 21.5 months $(95 \%$ CI, 17.2 to
25.8) in the AAw subgroup (Table 2, Fig. 1). The median OS was not reached in the other subgroups since more than half of the patients were still alive at the last follow-up. OS values for years 1-5 are presented in Table 2. PFS was 31.8 months (95\% CI, 17.6 to 46.2 ) for all patients, 16.4 months (95\% CI, 12.6 to 21.0$)$ in the AAw subgroup, and 130.0 months (95\% CI, 0.0 to 269.8) in the AOmc subgroup (Table 2, Fig. 2). PFS values for years 1-5 are presented in Table 2.

\section{Prognostic factors}

In univariate analysis, age, preoperative KPS score, tumor location, MGMT methylation status, postoperative residual tumor volume measured in contrast-enhanced T1-weighted MRI (RTV-T1CE), postoperative residual tumor volume measured in T2-weighted MRI (RTV-T2), EOR-T1CE, and EOR measured in T2-weighted MRI (EOR-T2) were statistically significant prognostic factors for OS and PFS (Table 3).

In the multivariate analysis, age, preoperative KPS, MGMT methylation status, RTV-T1CE, RTV-T2 and EOR-T2 were 
A
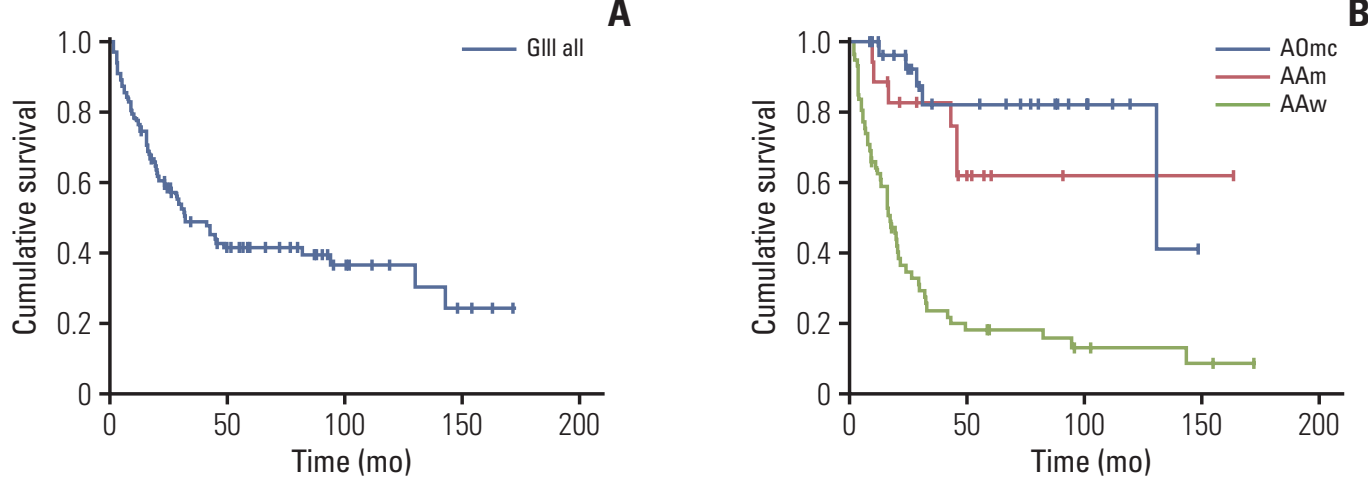

Fig. 2. Progression-free survival. (A) Kaplan-Meier representation of progression-free survival time for the entire group of 113 patients. (B) Kaplan-Meier representation of progression-free survival time for the AAw, AAm, AOmc each group. AAm, anaplastic astrocytoma, IDH-mutant; AAw, anaplastic astrocytoma, IDH-wildtype; AOmc, anaplastic oligodendroglioma, IDH-mutant and 1p/19q-codeleted; GIII, grade III glioma.

Table 3. Univariate analysis of prognostic factors

\begin{tabular}{|c|c|c|c|c|}
\hline & \multicolumn{2}{|l|}{ OS } & \multicolumn{2}{|l|}{ PFS } \\
\hline & HR $(95 \% \mathrm{CI})$ & p-value & $\mathrm{HR}(95 \% \mathrm{CI})$ & p-value \\
\hline Age & $1.025(1.008-1.042)$ & 0.004 & $1.022(1.006-1.038)$ & 0.008 \\
\hline Sex & $1.248(0.729-2.135)$ & 0.419 & $1.436(0.856-2.408)$ & 0.170 \\
\hline Preoperative KPS score & $0.911(0.866-0.958)$ & $<0.001$ & $0.932(0.893-0.972)$ & 0.001 \\
\hline Deep location & $0.333(0.193-0.577)$ & $<0.001$ & $0.316(0.186-0.537)$ & $<0.001$ \\
\hline MGMT methylation status & $0.405(0.236-0.694)$ & 0.001 & $0.418(0.251-0.696)$ & 0.001 \\
\hline Chemotherapy & $0.727(0.427-1.237)$ & 0.240 & $0.744(0.450-1.231)$ & 0.250 \\
\hline Preoperative T1CE tumor volume & $1.006(0.998-1.015)$ & 0.160 & $1.005(0.996-1.014)$ & 0.296 \\
\hline Preoperative T2 tumor volume & $0.995(0.990-1.000)$ & 0.073 & $0.995(0.991-1.000)$ & 0.057 \\
\hline Postoperative T1CE tumor volume & $1.155(1.085-1.229)$ & $<0.001$ & $1.119(1.058-1.184)$ & $<0.001$ \\
\hline Postoperative T2 tumor volume & $1.019(1.009-1.030)$ & $<0.001$ & $1.015(1.006-1.025)$ & 0.001 \\
\hline EOR (T1CE \%) & $0.985(0.973-0.997)$ & 0.013 & $0.986(0.974-0.998)$ & 0.018 \\
\hline EOR (T2 \%) & $0.976(0.964-0.987)$ & $<0.001$ & $0.977(0.966-0.988)$ & $<0.001$ \\
\hline
\end{tabular}

OS, overall survival; PFS, progression-free survival; HR, hazard ratio; CI, confidence interval; KPS, Karnofsky performance status; MGMT, $\mathrm{O}^{6}$-methylguanine-DNA methyltransferase; T1CE, T1-weighted contrast-enhanced magnetic resonance ima-ging (MRI); T2, T2-weighted MRI; EOR, extent of resection.

also statistically significant prognostic factor for OS, while age, MGMT methylation status, RTV-T1CE, RTV-T2, and EOR-T2 were statistically significant prognostic factors for PFS (Table 4).

\section{Cut-off value of EOR}

In cut-off value analysis using the Contal and O'Quigley method, age $<51$ years, complete resection of the enhanced portion $(99.96 \%)$, and more than $85.64 \%$ resection of the nonenhanced tumor portion showed prognostic impacts on OS in patients with anaplastic gliomas (Table 5, Fig. 3). As for PFS, age $<55$ years, $72.73 \%$ resection of the contrastenhanced portion, and $84.88 \%$ resection of the non-enhanced tumor portion demonstrated prognostic impacts (Table 5, Fig. 4).

\section{Discussion}

Despite multimodal treatment with surgery, radiotherapy, and chemotherapy, the prognosis for anaplastic glioma is poor. Several reports published in the past decade have shown survival times ranging from 19 months to 14.7 years for anaplastic gliomas [3,4,6-11]. Because of these varied prognoses, a new WHO 2016 classification, based on molecular markers, has been developed to promote more detailed and accurate diagnosis. Our study of 113 patients with anaplastic gliomas analyzed the survival, prognostic factors, and the cut-off value of extent of tumor resection, according to the 2016 WHO classification. Moreover, we found that the OS after surgery was 48.4 months for all anaplastic gliomas 
Table 4. Multivariate analysis of prognostic factors

\begin{tabular}{|c|c|c|c|c|}
\hline \multirow{2}{*}{ Variable } & \multicolumn{2}{|l|}{ Os } & \multicolumn{2}{|l|}{ PFS } \\
\hline & HR $(95 \%$ CI $)$ & p-value & HR $(95 \%$ CI $)$ & p-value \\
\hline Age & $1.068(1.023-1.115)$ & 0.003 & $1.073(1.029-1.118)$ & 0.001 \\
\hline Preop KPS & $0.889(0.805-0.981)$ & 0.019 & $0.921(0.847-1.001)$ & 0.052 \\
\hline Deep location & $0.302(0.083-1.101)$ & 0.070 & $0.334(0.104-1.077)$ & 0.066 \\
\hline MGMT methylation status & $0.150(0.037-0.613)$ & 0.008 & $0.092(0.022-0.381)$ & 0.001 \\
\hline Postoperative T1CE tumor volume & $1.301(1.056-1.602)$ & 0.013 & $1.335(1.071-1.663)$ & 0.010 \\
\hline Postoperative T2 tumor volume & $1.054(1.009-1.100)$ & 0.019 & $1.076(1.027-1.129)$ & 0.002 \\
\hline EOR (T1CE \%) & $0.999(0.975-1.024)$ & 0.925 & $0.993(0.969-1.017)$ & 0.578 \\
\hline $\mathrm{EOR}(\mathrm{T} 2 \%)$ & $0.951(0.915-0.988)$ & 0.010 & $0.942(0.907-0.978)$ & 0.002 \\
\hline
\end{tabular}

OS, overall survival; PFS, progression-free survival; HR, hazard ratio; CI, confidence interval; KPS, Karnofsky performance status; MGMT, $\mathrm{O}^{6}$-methylguanine-DNA methyltransferase; T1CE, T1-weighted contrast-enhanced magnetic resonance imaging (MRI); T2, T2-weighted MRI; EOR, extent of resection.

Table 5. Cut-off point (Contal and O'Quigley method)

\begin{tabular}{|c|c|c|c|c|c|c|}
\hline \multirow{2}{*}{ Variable } & \multicolumn{3}{|c|}{ OS } & \multicolumn{3}{|c|}{ PFS } \\
\hline & Cut point & HR $(95 \%$ CI $)$ & p-value & Cut point & HR $(95 \%$ CI $)$ & p-value \\
\hline Age & $\geq 51$ & $2.911(1.880-4.508)$ & $<0.001$ & $\geq 55$ & $2.971(1.942-4.545)$ & $<0.001$ \\
\hline EOR (T1CE \%) & $\geq 99.957$ & $0.284(0.154-0.524)$ & $<0.001$ & $\geq 72.727$ & $0.370(0.227-0.602)$ & $<0.001$ \\
\hline $\mathrm{EOR}(\mathrm{T} 2 \%)$ & $\geq 85.643$ & $0.141(0.075-0.267)$ & $<0.001$ & $\geq 84.883$ & $0.193(0.112-0.331)$ & $<0.001$ \\
\hline
\end{tabular}

OS, overall survival; PFS, progression-free survival; HR, hazard ratio; CI, confidence interval; EOR, extent of resection; T1CE, T1-weighted contrast-enhanced magnetic resonance imaging (MRI); T2, T2-weighted MRI.

and 21.5 months in the AAw subgroup (Table 2).

Previously reported prognostic factors for anaplastic glioma include advanced patient age, preoperative neurological status, KPS, symptom duration, tumor location, EOR, adjuvant therapy (including radiation therapy and chemotherapy), preoperative MRI findings, as well as various molecular markers including IDH and PTEN mutations, $1 \mathrm{p} / 19 \mathrm{q}$ codeletion, epidermal growth factor receptor (EGFR) amplification, and MGMT methylation [4,7-9,12-15]. The importance of these molecular markers for prognosis was reflected in the new WHO classification of 2016, which has had a great impact on the diagnostic criteria. Here, we have confirmed several prognostic factors including age, preoperative KPS, MGMT methylation status, postoperative tumor volume, and EOR.

Aggressive tumor resection can be dangerous for the patient's neurologic function, especially when the tumor is located deep inside the brain. Thus, when evaluating the association between survival and the EOR, it is important to take the tumor's location into account. In our series, tumor location was a statistically significant prognostic factor in univariate analysis, but its effect was lost in multivariate analysis. In addition, the association between preoperative tumor volume and survival rate was not statistically significant. These results suggest that the degree of surgical removal has a greater effect on the prognosis than the preoperative size and location.

In our univariate analysis, postoperative residual tumor volume and EOR were statistically significant prognostic factors for OS and PFS. This suggests that the extent of surgical resection and remaining tumor volume after surgery may have greater impacts on prognosis than preoperative volume (Table 3). However, in multivariate analysis, RTV-T1CE, RTV-T2, and EOR-T2 were statistically important prognostic factors (Table 4). The statistical insignificance of EOR-T1CE in multivariate analysis appears to be an effect of T2 lesions. Basically, T1 contrast-enhanced lesions are included in T2 lesions. Therefore, as shown in previous studies, we hypothesize that the EOR-T1CE still has significant prognostic value, and a study of the EOR cut-off value can be performed.

Malignant astrocytomas, including anaplastic glioma and glioblastoma multiforme, are difficult to resect curatively because of their invasive and infiltrative nature to the surrounding tissue [16]. This is especially difficult if the tumor is located in a functionally important region. However, microsurgical resection is a very important factor in the treatment of glioma, and maximal safe resection is known to be a good prognostic factor for all grades of gliomas [17-22].

In past, some studies had reported that there is no relationship between the EOR and survival in anaplastic gliomas 
A
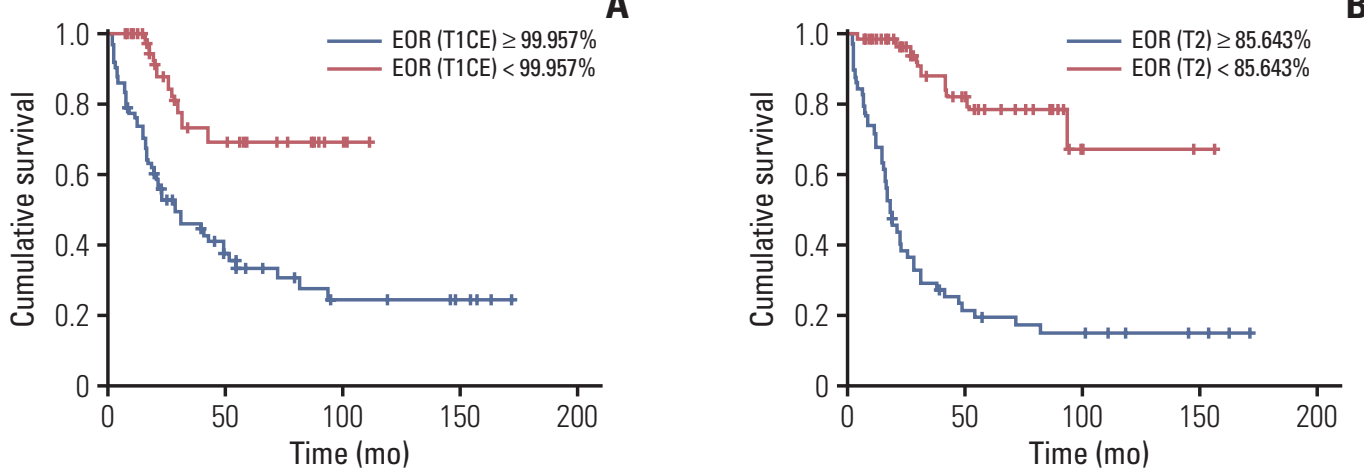

Fig. 3. Overall survival. (A) Kaplan-Meier representation of overall survival time according to EOR (T1CE). (B) Kaplan-Meier representation of overall survival time according to EOR (T2). EOR, extent of resection; T1CE, T1-weighted contrast-enhanced magnetic resonance imaging (MRI); T2, T2-weighted MRI.

A



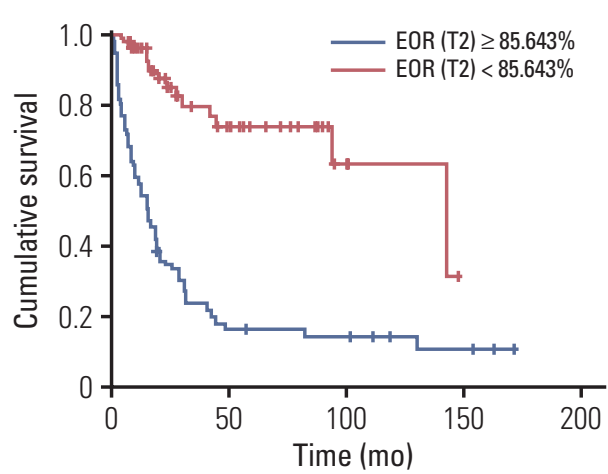

B

Fig. 4. Progression-free survival. (A) Kaplan-Meier representation of progression-free survival time according to EOR (T1CE). (B) KaplanMeier representation of progression-free survival time according to EOR (T2). EOR, extent of resection; T1CE, T1-weighted contrast-enhanced magnetic resonance imaging (MRI); T2, T2-weighted MRI.

$[23,24]$. However, recent studies have revealed that there is a relationship between the EOR and survival in anaplastic gliomas $[3,25,26]$. We performed volumetric analysis in the present study, confined to anaplastic gliomas, which have heterogenous features in MRI. A considerable proportion of anaplastic gliomas do not show contrast enhancement in T1weighted MRI $[15,17]$. Therefore, to obtain a more accurate tumorvolume, both abnormalT2/FLAIRhyperintenselesions as well as T1-weighted contrast-enhanced lesions should be considered. Previous studies analyzed EOR by combining tumor volume measured in T1-weighted contrast-enhanced images with that of T2/FLAIR image or T2 image alone $[25,26]$. To obtain more accurate information, we analyzed T2-weighted and T1 contrast-enhanced images separately and attempted to determine whether the EOR of each sequence affects survival rate.

Previous studies have found EOR thresholds of $76 \%$ in GIII glioma, 90\% in GII glioma, 100\% contrast enhancing resection with additional $53.21 \%$ of FLAIR hyperintense lesion in glioblastoma, and 53\% in anaplastic astrocytoma and anaplastic oligoastrocytoma $[18,22,25,26]$.

We investigated the cut-off value for the EOR on each MRI sequence (Table 5). The cut-off value of EOR affecting OS was 99.96\% in contrast-enhanced T1-weighted MRI and 85.64\% in T2-weighted MRI, so we propose that these EOR values are important for anaplastic gliomas. The use of multiple MRI sequences for suggesting the cut-off value of the volumetric EOR represents a strength of our study in the era of molecular glioma classification.

Age has already been identified as an important prognostic factor in several studies $[3,7,24]$. For example, a study has reported that an age of 65 years or older is a poor prognostic factor [27]. In the present study, we confirmed that age is an important factor affecting survival: 51 years old was the cutoff value that influenced the OS rate, while a cut-off of 55 years affected PFS.

Our study has some limitations. First, because of its retrospective nature from a single institution, there may be a selection bias of the patients. A few cases were excluded because of inadequate information of MRI available for 
review. However, we tried to analyze a uniform patient population by examining consecutive patients. Second, there may be measuring bias. Because we measured T2-weighted hyperintense lesions separately, cerebral edema, ischemic change, and contusions may have been included to tumor volume in some degree. Third, patients have received various chemotherapeutic agents although the regimens of chemotherapy did not have a statistical significance in univariate analysis. And the postoperative radiation therapy was not controlled. In the future, more controlled multicenter validation studies are required.

In conclusion, the median OS was 48.4 months in the whole anaplastic glioma group and 21.5 months in the AAw group. We have also revealed that complete resection (more than 99.96\%) of tumor volume measured in contrast-enhanced
T1-weighted MRI, and more than $85.64 \%$ of tumor resection measured in T2-weighted MRI, have prognostic impacts on the survival of patients with anaplastic gliomas. Therefore, gross-total resection of at least the contrast-enhanced part of a lesion should be performed to prolong survival in anaplastic glioma patients.

\section{Conflict of Interest}

Conflict of interest relevant to this article was not reported.

\section{Acknowledgments}

This study was supported by the National Research Foundation of Korea (NRF) grant funded by the Korean government (NRF2014R1A1A2058058) and a faculty research grant of Yonsei University College of Medicine (6-2018-0061).

\section{References}

1. Ostrom QT, Gittleman H, Fulop J, Liu M, Blanda R, Kromer $\mathrm{C}$, et al. CBTRUS statistical report: primary brain and central nervous system tumors diagnosed in the United States in 2008-2012. Neuro Oncol. 2015;17 Suppl 4:iv1-62.

2. Louis DN, Perry A, Reifenberger G, von Deimling A, FigarellaBranger D, Cavenee WK, et al. The 2016 World Health Organization classification of tumors of the central nervous system: a summary. Acta Neuropathol. 2016;131:803-20.

3. Nuno M, Birch K, Mukherjee D, Sarmiento JM, Black KL, Patil CG. Survival and prognostic factors of anaplastic gliomas. Neurosurgery. 2013;73:458-65.

4. Rogne SG, Konglund A, Scheie D, Helseth E, Meling TR. Anaplastic astrocytomas: survival and prognostic factors in a surgical series. Acta Neurochir (Wien). 2014;156:1053-61.

5. Contal C, O'Quigley J. An application of changepoint methods in studying the effect of age on survival in breast cancer. Comput Stat Data Anal. 1999;30:253-70.

6. Cairncross G, Wang M, Shaw E, Jenkins R, Brachman D, Buckner J, et al. Phase III trial of chemoradiotherapy for anaplastic oligodendroglioma: long-term results of RTOG 9402. J Clin Oncol. 2013;31:337-43.

7. Chaichana KL, Kosztowski T, Niranjan A, Olivi A, Weingart JD, Laterra J, et al. Prognostic significance of contrast-enhancing anaplastic astrocytomas in adults. J Neurosurg. 2010;113: 286-92.

8. van den Bent MJ, Brandes AA, Taphoorn MJ, Kros JM, Kouwenhoven MC, Delattre JY, et al. Adjuvant procarbazine, lomustine, and vincristine chemotherapy in newly diagnosed anaplastic oligodendroglioma: long-term follow-up of EORTC brain tumor group study 26951. J Clin Oncol. 2013;31:344-50.

9. Wick W, Hartmann C, Engel C, Stoffels M, Felsberg J, Stockhammer $\mathrm{F}$, et al. NOA-04 randomized phase III trial of sequential radiochemotherapy of anaplastic glioma with procarbazine, lomustine, and vincristine or temozolomide. J Clin Oncol. 2009;27:5874-80.10.

10. Wick W, Roth P, Hartmann C, Hau P, Nakamura M, Stock- hammer F, et al. Long-term analysis of the NOA-04 randomized phase III trial of sequential radiochemotherapy of anaplastic glioma with PCV or temozolomide. Neuro Oncol. 2016;18:1529-37.

11. Hildebrand J, Gorlia T, Kros JM, Afra D, Frenay M, Omuro A, et al. Adjuvant dibromodulcitol and BCNU chemotherapy in anaplastic astrocytoma: results of a randomised European Organisation for Research and Treatment of Cancer phase III study (EORTC study 26882). Eur J Cancer. 2008;44:1210-6.

12. Hartmann C, Hentschel B, Wick W, Capper D, Felsberg J, Simon M, et al. Patients with IDH1 wild type anaplastic astrocytomas exhibit worse prognosis than IDH1-mutated glioblastomas, and IDH1 mutation status accounts for the unfavorable prognostic effect of higher age: implications for classification of gliomas. Acta Neuropathol. 2010;120:707-18.

13. Paleologos NA, Merrell RT. Anaplastic glioma. Curr Treat Options Neurol. 2012;14:381-90.

14. Stupp R, Reni M, Gatta G, Mazza E, Vecht C. Anaplastic astrocytoma in adults. Crit Rev Oncol Hematol. 2007;63:72-80.

15. Wang Y, Wang K, Wang J, Li S, Ma J, Dai J, et al. Identifying the association between contrast enhancement pattern, surgical resection, and prognosis in anaplastic glioma patients. Neuroradiology. 2016;58:367-74.

16. Claes A, Idema AJ, Wesseling P. Diffuse glioma growth: a guerilla war. Acta Neuropathol. 2007;114:443-58.

17. Keles GE, Chang EF, Lamborn KR, Tihan T, Chang CJ, Chang $\mathrm{SM}$, et al. Volumetric extent of resection and residual contrast enhancement on initial surgery as predictors of outcome in adult patients with hemispheric anaplastic astrocytoma. J Neurosurg. 2006;105:34-40.

18. Li YM, Suki D, Hess K, Sawaya R. The influence of maximum safe resection of glioblastoma on survival in 1229 patients: can we do better than gross-total resection? J Neurosurg. 2016;124: 977-88.

19. McGirt MJ, Chaichana KL, Gathinji M, Attenello FJ, Than K, Olivi A, et al. Independent association of extent of resection 
with survival in patients with malignant brain astrocytoma. J Neurosurg. 2009;110:156-62.

20. Nitta M, Muragaki Y, Maruyama T, Ikuta S, Komori T, Maebayashi K, et al. Proposed therapeutic strategy for adult lowgrade glioma based on aggressive tumor resection. Neurosurg Focus. 2015;38:E7.

21. Sanai N, Polley MY, McDermott MW, Parsa AT, Berger MS. An extent of resection threshold for newly diagnosed glioblastomas. J Neurosurg. 2011;115:3-8.

22. Smith JS, Chang EF, Lamborn KR, Chang SM, Prados MD, Cha $\mathrm{S}$, et al. Role of extent of resection in the long-term outcome of low-grade hemispheric gliomas. J Clin Oncol. 2008;26:1338-45.

23. Devaux BC, O'Fallon JR, Kelly PJ. Resection, biopsy, and survival in malignant glial neoplasms: a retrospective study of clinical parameters, therapy, and outcome. J Neurosurg. 1993; 78:767-75.

24. Tortosa A, Vinolas N, Villa S, Verger E, Gil JM, Brell M, et al.
Prognostic implication of clinical, radiologic, and pathologic features in patients with anaplastic gliomas. Cancer. 2003;97: 1063-71.

25. Fujii Y, Muragaki Y, Maruyama T, Nitta M, Saito T, Ikuta S, et al. Threshold of the extent of resection for WHO grade III gliomas: retrospective volumetric analysis of 122 cases using intraoperative MRI. J Neurosurg. 2018;129:1-9.

26. Pessina F, Navarria P, Cozzi L, Ascolese AM, Simonelli M, Santoro A, et al. Value of surgical resection in patients with newly diagnosed grade III glioma treated in a multimodal approach: surgery, chemotherapy and radiotherapy. Ann Surg Oncol. 2016;23:3040-6.

27. Tanaka S, Meyer FB, Buckner JC, Uhm JH, Yan ES, Parney IF. Presentation, management, and outcome of elderly patients with newly-diagnosed anaplastic astrocytoma. J Neurooncol. 2012;110:227-35. 